\title{
MOUNTAINOUS REMOTE SENSING IMAGES REGISTRATION BASED ON IMPROVED OPTICAL FLOW ESTIMATION
}

\author{
Ruitao Feng ${ }^{1}$, Xinghua $\mathrm{Li}^{2}$, Huanfeng Shen ${ }^{1,3,4, *}$ \\ ${ }^{1}$ School of Resource and Environmental Sciences, Wuhan University, P. R. China - (ruitaofeng, shenhf)@ whu.edu.cn \\ ${ }^{2}$ School of Remote Sensing and Information Engineering, Wuhan University, P. R. China - lixinghua5540@whu.edu.cn \\ ${ }^{3}$ Collaborative Innovation Center of Geospatial Technology, Wuhan University, P. R. China \\ ${ }^{4}$ Key Laboratory of Geographic Information System, Ministry of Education, Wuhan University, P. R. China
}

KEY WORDS: Displacement Modification, Laplacian of Gaussian, Mountainous Remote Sensing Image, Optical Flow, Registration

\begin{abstract}
:
Mountainous remote sensing images registration is more complicated than in other areas as geometric distortion caused by topographic relief, which could not be precisely achieved via constructing local mapping functions in the feature-based framework. Optical flow algorithm estimating motion of consecutive frames in computer vision pixel by pixel is introduced for mountainous remote sensing images registration. However, it is sensitive to land cover changes that are inevitable for remote sensing image, resulting in incorrect displacement. To address this problem, we proposed an improved optical flow estimation concentrated on postprocessing, namely displacement modification. First of all, the Laplacian of Gaussian (LoG) algorithm is employed to detect the abnormal value in color map of displacement. Then, the abnormal displacement is recalculated in the interpolation surface constructed by the rest accurate displacements. Following the successful coordinate transformation and resampling, the registration outcome is generated. Experiments demonstrated that the proposed method is insensitive in changeable region of mountainous remote sensing image, generating precise registration, outperforming the other local transformation model estimation methods in both visual judgment and quantitative evaluation.
\end{abstract}

\section{INTRODUCTION}

Image registration is an alignment processing, which matches two or more images taken by the same or different sensors from different periods, the same/different angles of view about the same scene (Wong and Clausi 2007). As a fundamental preprocessing, it plays a significant role in remote sensing image applications, like image fusion (Shen et al. 2016), image mosaic (Li et al. 2015), multi-temporal image analysis (Zeng et al. 2013) and so forth. The conventional registration algorithms are always classified into two categories, including feature- and area-based algorithm.

The study on area-based method mainly focuses on similarity metric construction, such as cross correlation (CC) (Martinez et al. 1999), the mutual information (MI) (Johnson et al. 2001), the normalized cross coefficients (NCC) (Hel-Or et al. 2011), etc. These methods make full use of the intensity information and achieve high registration accuracy whereas they have weak ability to handle large deformation between reference and sensed image. As for the feature-based method, more attention is put on the feature extraction and mismatched feature elimination. Taking the feature point as an example, Uniform robust SIFT (UR-SIFT) (Sedaghat et al. 2011), scale restrict SIFT (SR-SIFT) (Yi et al. 2008), speed-up robust feature (SURF) (Bay et al. 2006), KAZE (Alcantarilla et al. 2012) etc. are proposed gradually to obtain robust feature points. With the matched feature points, the random sample consensus (RANSAC) (Fischler and Bolles 1981), maximum likelihood estimation sample consensus (MLESAC) (Wang et al. 2010) or other algorithms are employed to remove the mismatched for accurate transformation model design. The feature-based method registers images with extracted features rather than the original intensity information. Therefore, it is time-saving.

With accurate matched intensity information or features, it is necessary to design precise geometrical relationship for reference and sensed image. Besides the traditional global model, some local models like piecewise linear mapping function (PLM) (Goshtasby 1986), weighted mean (WM) , multiquadric (MQ) (Zagorchev and Goshtasby 2006) and block weighted projective (BWP) model (Feng et al. 2017) etc. are adopted. However, their accuracy depends on the distribution, the number and the position precision of the feature points (Zagorchev and Goshtasby 2006). It is difficult to extract ideal feature points for local geometrical mapping function construction in mountain area. And we find that the per-pixel registration is more suitable for inconsistent distortion everywhere of mountainous remote sensing image. As the typical representation of per-pixel registration algorithm, optical flow algorithm directly utilizes pixel itself information under global constraints, estimating sub-pixel displacement pixel by pixel.

The optical flow algorithm is popular with computer vision image process, which was first mentioned in 1950s and the calculation algorithm was firstly proposed by Horn and Schunch (Horn and Schunck 1981). For accurate optical flow estimation, the modified penalty function according to the robust statistics for outliers and multi motions was proposed in 1996 (Black and Anandan 1996). Liu et al. proposed describing images with SIFT descriptors instead of the original intensity

\footnotetext{
* Corresponding author
} 
information for robust optical flow estimation (Liu et al. 2011). Ren et al. combines homography transformation model globally with L-K optical flow to construct mesh flow (Ren et al. 2017), and Lin et al. extends the algorithm for photograph registration (Lin et al. 2017). Inspired by it, we introduce the optical flow algorithm to mountainous remote sensing images registration. However, seasonal change, geological disaster, construction and demolition of buildings etc. always lead to ground objects inconsistency, weakening the optical flow algorithm and resulting in incorrect displacement. In terms of this issue, we propose a post-processing, namely displacement modification. Firstly, the incorrect displacement is detected by Laplacian of Gaussian (LoG). And then, it is modified with curve modeling and interpolation.

The rest of the paper is organized as follows. In Section 2, we give a description of registration algorithm, in which the attention is put on the mask calculation for abnormal displacement and displacement modification. After that, we verify the proposed method by real data in Section 3. At last, a conclusion for the current work and the plan for future work are provided in Section 4.

\section{METHODOLOGY}

Optical flow estimation is employed for mountainous remote sensing images registration in this paper. However, with inevitable land cover changes, it performs poorly to accurately estimate deformation in changeable regions. Towards this problem, we propose the displacement modification algorithm for optical flow estimation post-processing. The process of mountainous remote sensing images registration is represented as follow.

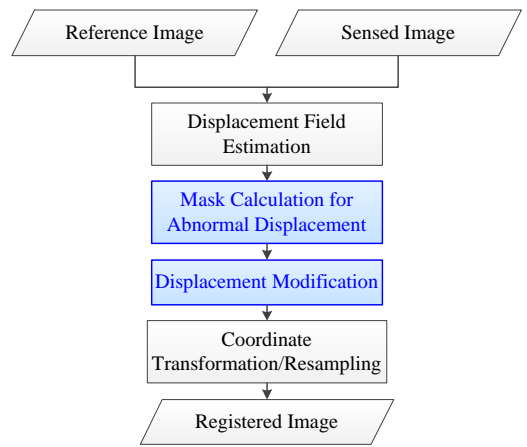

Figure 1. Mountainous remote sensing image registration.

Displacements estimation is equivalent to the calculation of deformation between reference and sensed image. The typical optical flow estimation algorithm is employed as expressed in
Eq.(1) (Brox et al. 2004).

$$
\begin{aligned}
E(u, v)= & \int_{\Omega} \psi\left(|I(X+W)-I(X)|^{2}\right) d X \\
& +\gamma \int_{\Omega} \psi\left(|\nabla I(X+W)-\nabla I(X)|^{2}\right) d X \\
& +\alpha \int_{\Omega} \psi\left(\left|\nabla_{3} u\right|^{2}+\left|\nabla_{3} v\right|^{2}\right) d X
\end{aligned}
$$

where $W=(u, v, 1)^{T}$ is the displacement field and $X=(x, y, 1)^{T}$ is the pixel coordinate. $\psi\left(s^{2}\right)=\sqrt{s^{2}+\varepsilon^{2}}$ is the penalty function. $\nabla_{3}:=\left(\partial_{x}, \partial_{y}, \partial_{t}\right)^{T}$ indicates a spatio-temporal gradient, which is replaced by the spatial gradient in our experiment. $\gamma$ and $\alpha$ are weight, set as 5 and 80 respectively in the experiment.

As seen, this model combines the intensity ans gradient information, which is more robust to radiation difference and large displacement. However, the calculated displacement in changeable area is always not accurate as there is no corresponding pixel in another image. Under this circumstance, it is useful to supply it with a post-processing, which is to describe in detail at the following section.

\subsection{Mask calculation for abnormal displacement}

Land cover changes always generate non-correspondence between reference and sensed image. It causes inaccurate deformation estimation, resulting in content loss compared with original sensed image. For example, Figure 2.(a)-(b) are original reference and sensed image, and Figure 2.(c) is the aligned image. As seen, the road locating in the center of Figure 2.(c) is different from that of in Figure 2.(b). While focusing on the same place in Figure 2.(a), it is different from the sensed image either. The non-correspondence between reference and sensed image generates the incorrect displacement resulting in difference between aligned image and the sensed image [see Figure 2.(b) and Figure 2.(c)].

To detect the abnormal displacement region, it is difficult to discover the rule through observing the displacement directly. If we represent the original displacement estimated by optical flow algorithm in Munsell color system in Figure 2.(d), it is easy to observe. In Figure 2.(d), different colors indicate different directions of displacement and depth represents the magnitude of it. As seen, region with rich colors indicates the disunity direction of displacement, corresponding to varying surface of images [see Figure 2. (a)-(d)]. Therefore, calculating abnormal displacement mask is transferred into detecting obvious color mutation regions.

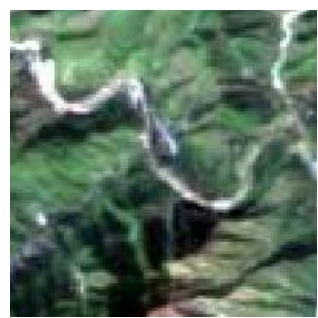

(a)

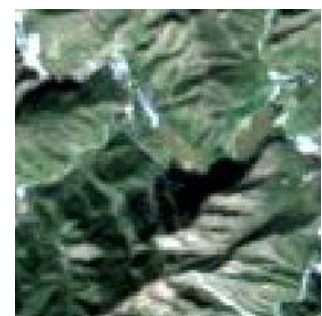

(b)

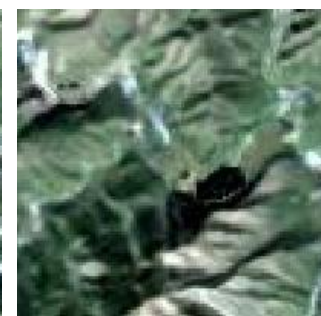

(c)

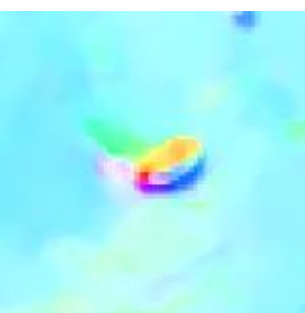

(d)

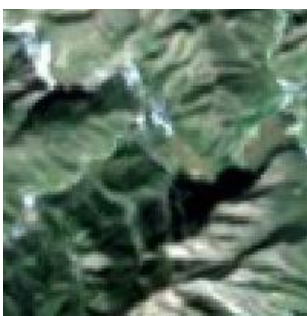

(e)

Figure 2. Registration result with optical flow algorithm. (a) Reference image. (b) Sensed image. (c) Registered image without postprocessing (Brox et al. 2004). (d) Color map of displacement. (e) Registered image with displacement post-processing. 
In the color map, the color mutation region is similar to blob, which is detected by Laplacian of Gaussian (LoG) algorithm generally and widely in multi scales. As known, Gaussian function is expressed as Eq.(2).

$$
G(x, y ; \sigma)=\frac{1}{2 \pi \sigma^{2}} e^{-\frac{\left(x^{2}+y^{2}\right)}{\sigma^{2}}}
$$

And LoG is the sum of the second partial derivative of Gaussian function in $\mathrm{x}$ and $\mathrm{y}$ direction, which could be described as Eq.(3).

$$
\nabla^{2} G=\frac{\partial^{2} G}{\partial x^{2}}+\frac{\partial^{2} G}{\partial y^{2}}
$$

We employed the normalized LoG convolve with the color map of original displacement to obtain the mask.

$$
\begin{aligned}
L(x, y ; \sigma) & =\nabla_{\text {norm }}^{2} * f(x, y)=\sigma^{2} \nabla^{2} G \\
& =\frac{1}{2 \pi \sigma^{2}}\left[1-\frac{x^{2}+y^{2}}{\sigma^{2}}\right] \cdot e^{-\frac{x^{2}+y^{2}}{2 \sigma^{2}}}
\end{aligned}
$$

where $\sigma$ is the scale factor and $(x, y)$ is image coordinate.

With the detected blobs in the color map, the mask of the abnormal displacement is constructed. And then the abnormal displacement labeled by the mask is modified.

\subsection{Displacement Modification}

Except the abnormal displacement in changeable area, the rest displacements are relatively accurate. Therefore, we utilize them to create interpolated surface as shown in Figure 3, in which the colorful grid is the interpolated surface. With the position of abnormal displacement, labeled in red circles in Figure 3, we recalculate the displacement with bilinear interpolation in the surface.

$$
\begin{aligned}
f(i+u, j+v) & =(1-u)(1-v) f(i, j)+(1-u) v f(i, j+1) \\
& +u(1-v) f(i+1, j)+u v f(i+1, j+1)
\end{aligned}
$$

where $(i+u, j+v)$ is coordinate of the abnormal displacement, and $(u, v)$ is the decimal part of the coordinate. $f(*)$ denotes the displacement.

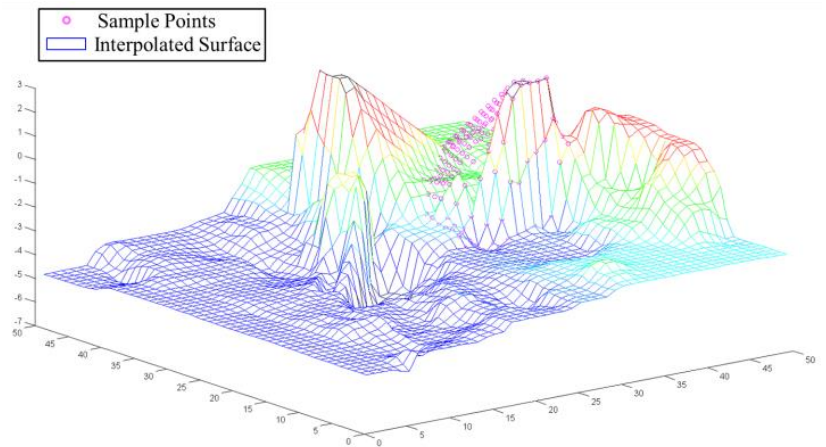

Figure 3. The interpolated surface and abnormal displacement position

Handling the displacement with a post-processing, only the geometric position of the original sensed image is changed to align to the reference image and the surface of aligned image is consistent with the original sensed image as in Figure 2.(e).

\section{EXPERIMENTS}

In this paper, experimental images are clipped from the level$1 \mathrm{~A}$ data with the process of homogenized radiation calibration from the wide field of view (WFV) imaging system onboard the Chinese GF-1 optical satellite. The spatial resolution is $16 \mathrm{~m}$ and the width is $800 \mathrm{~km}$. There are inconsistent geometrical distortions especially in mountainous area. In addition to this, land cover changes and radiation difference are visible everywhere.

Experiment 1: The reference image is taken on February 12, 2017 and the source image is taken on August 28, 2016, showed in Figure 4.(a)-(b). The experimental region locates at Qinling mountain, Shannxi province, China. The size of image is $500 * 500$. There is seasonal change, leading to radiation difference and land cover changes.

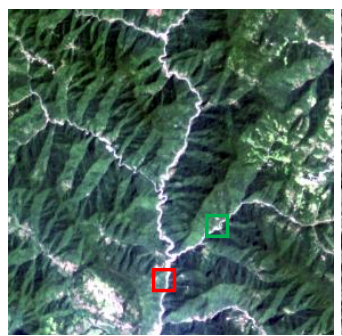

(a)

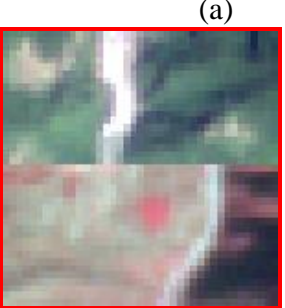

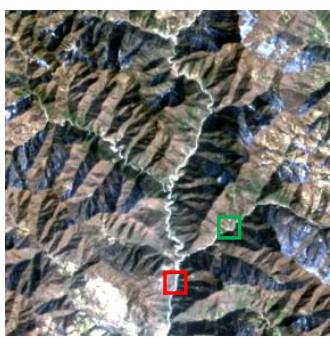

(b)

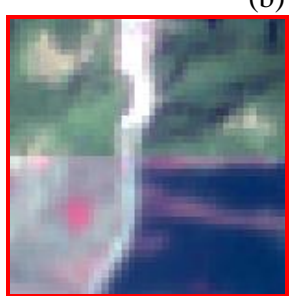

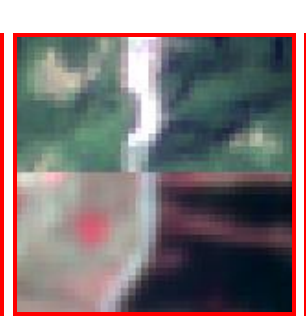

(c)

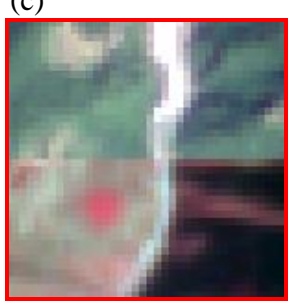

(d)

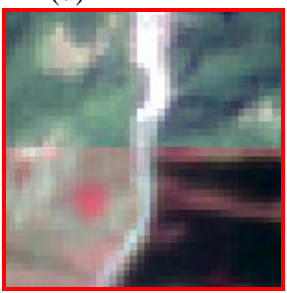




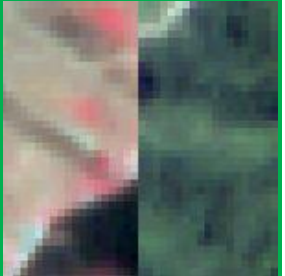

(e)

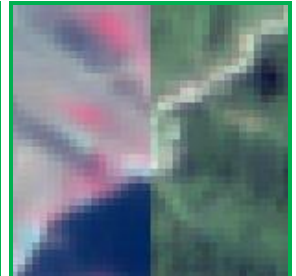

(f)

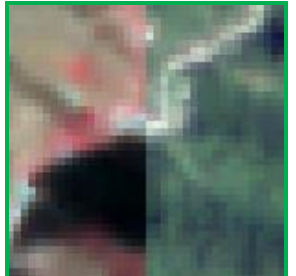

(g)

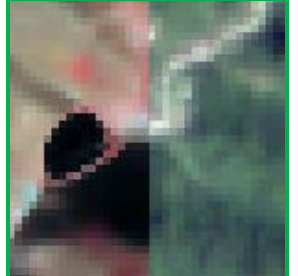

(h)

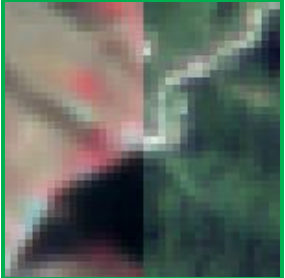

(i)

Figure 4. The first experiment. (a) Reference image. (b) Sensed image. (c) Overlapping of the original images. (d) Overlapping of the reference and aligned image from the proposed method. Magnified images of selected area marked in red and green rectangles from (e) the original images, (f) PLM (Goshtasby 1986), (g) BWP (Feng et al. 2017), (h) optical flow without post-processing (Brox et al. 2004), and (i) The proposed method (DM_OF).

Experiment 2: The reference image is taken on July 08, 2017 and the sensed image is taken on September 20, 2017, showed in Figure 5.(a)-(b). The size of the experimental image is

$400 * 400$. The image covers the northwestern of Beijing, China. There is radiation difference as the reference image is taken in summer but the sensed image is taken in autumn.

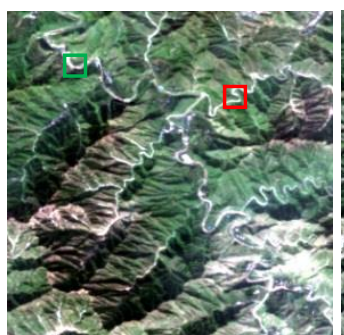

(a)
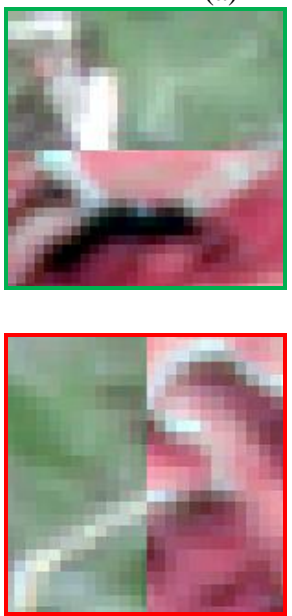

(e)

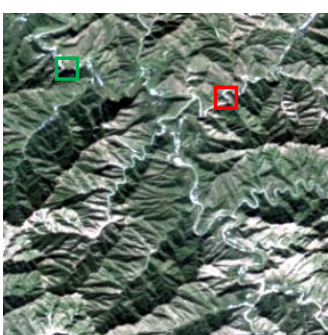

(b)
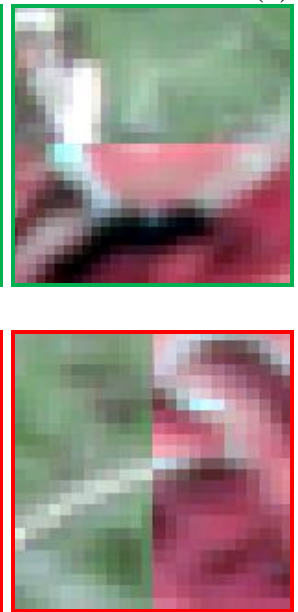

(f)

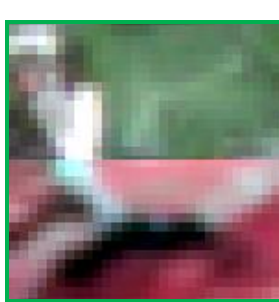

(c)
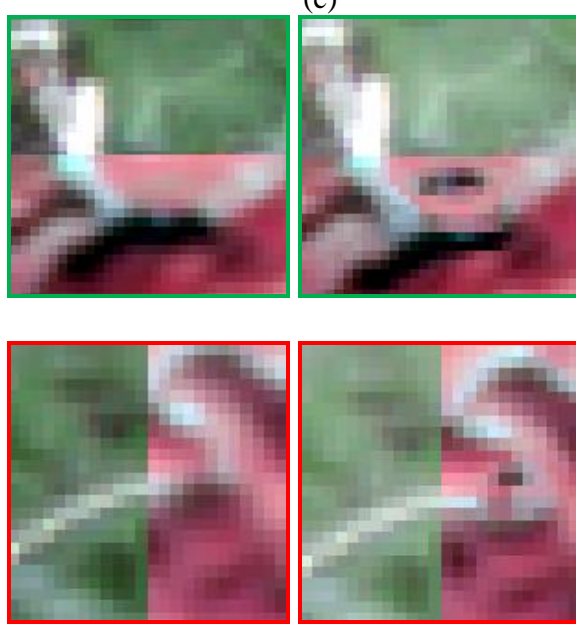

(g)

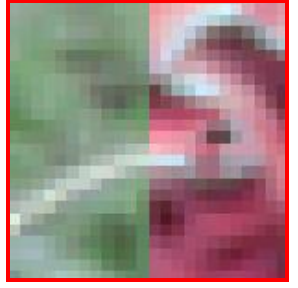

(h)

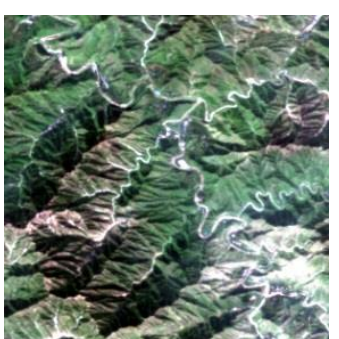

(d)
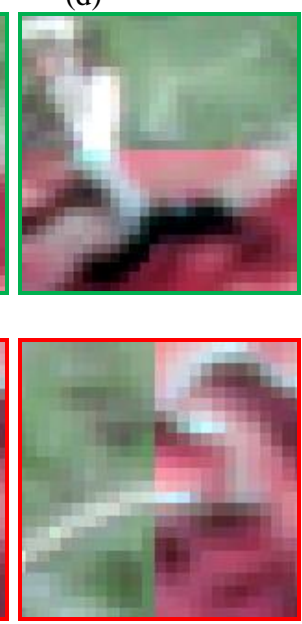

(i)

Figure 5. The second experiment. (a) Reference image. (b) Sensed image. (c) Overlapping of the original images. (d) Overlapping of the reference and aligned image from the proposed method. Magnified images of selected area marked in red and green rectangles from (e) the original images, (f) PLM (Goshtasby 1986), (g) BWP (Feng et al. 2017), (h) optical flow without post-processing (Brox et al. 2004), and (i) the proposed method (DM_OF).

For both Figure 4 and Figure 5, (a) and (b) are the original reference and sensed image, respectively. Figure 4.(c) and Figure 5.(c) are the overlapping of the original images, where the distinct ghosts resulting from the misalignment vanished and they looked a lot clearer. In order to observe the results detailedly and compare the outcomes of different algorithms, the selected region marked in red and green rectangle of corresponding images are zoomed in as showed in Figure 4.(e)(h) and Figure 5.(e)-(h). As seen, the reference image is shown in true color and the original sensed image and the registration results are shown in pseudo color (the NIR, red, and green bands are assigned to the red, green, and blue channels, respectively). In Figure 4 and Figure 5, (e) is selected and zoomed in from the original images. (f) is the result from PLM and $(\mathrm{g})$ is from the BWP. (h) is the result of optical flow algorithm without post-processing and (i) is the result by proposed method. As seen, the proposed algorithm and the optical flow algorithm without post-processing outperform others both in horizontal and vertical direction. However, the content in Figure 4(h) is abnormal comparing with the original sensed image where there is a black hole in aligned image processed without post-processing. The same conclusion could be drawn in Figure 5.(h) even though the accurate registration is obtained. The contents in Figure 4(i) and Figure 5.(i) are consistent with the original sensed image. Comparing the geometrical registration, BWP (Feng et al. 2017) performs better than PLM (Goshtasby 1986), which couldn't eliminate the deformation between reference and sensed image thoroughly[see Figure 4(f) and Figure 5.(f)]. BWP achieve vertical alignment in Figure 4(h) and Figure 5.(h) while 
focusing on the enlarged image in vertical direction at the bottom. However, BWP couldn't guarantee good registration results everywhere. Therefore, the proposed method containing post-processing of optical flow algorithm shows the best performance on the whole.
Besides the visual judgement, three evaluation indicators are employed such as normalized mutual information (NMI), correlation coefficients (CC) and root mean square error (RMSE), to evaluate the registration quantitatively. For brevity, the proposed method is abbreviated as "DM_OF" in Table 1.

Table 1. Quantitative evaluation of the experiments

\begin{tabular}{c|cccc}
\hline \hline & & NMI $(\uparrow)$ & CC $(\uparrow)$ & RMSE $(\downarrow)$ \\
\hline \multirow{5}{*}{ Experiment 1 } & Original & 1.0274 & 0.3594 & 17.935 \\
& PLM & 1.0665 & 0.7397 & 1.6013 \\
& BWP & 1.0683 & 0.7439 & 1.4887 \\
& DM_OF & $\mathbf{1 . 0 7 5 6}$ & $\mathbf{0 . 7 8 1 0}$ & $\mathbf{0 . 8 7 2 2}$ \\
\hline \multirow{3}{*}{ Experiment 2 } & Original & 1.0578 & 0.6167 & 4.3304 \\
& PLM & 1.0893 & 0.8395 & 1.2313 \\
& BWP & 1.0938 & 0.8534 & 1.0237 \\
& DM_OF & $\mathbf{1 . 1 0 4 5}$ & $\mathbf{0 . 8 8 8 3}$ & $\mathbf{0 . 2 1 9 1}$ \\
\hline \hline
\end{tabular}

(For brevity, 'Original' represents the deformation between original reference and sensed image, and the proposed method is abbreviated as 'DM_OF'.

The available range of NMI is one to two and the available range of $\mathrm{CC}$ is minus one to one. Large NMI and $\mathrm{CC}$ represent the satisfied registration result. The smaller RMSE, the better outcome. Especially, we did not give quantitative evaluation of the registration result without post-processing. As shown in Table 1 , the original deformations both in the first and second experiment are large and all the methods could alleviate them. Furthermore, the same conclusion could be drawn in the two experiments. The BWP performs better than the PLM algorithm. And the proposed method outperforms others, which has the highest NMI and CC, and the RMSE is the smallest one.

In a word, the proposed method outperforms others in mountainous remote sensing images registration without content inconsistency comparing with original sensed image both in the term of visual judgement and quantitative evaluation.

\section{CONCLUSION}

To satisfy high-precision registration of mountainous remote sensing images, the optical flow algorithm is employed, estimating the displacement pixel by pixel. Aiming at the sensibility of optical flow method in changeable area, this paper proposed an improved optical flow algorithm, namely displacement modification. The abnormal displacement is detected by LoG and corrected in interpolation surface constructed with the accurate displacements. The proposed algorithm registers mountainous remote sensing image precisely while the content is consistent with original sensed image. However, the proposed algorithm mainly focuses on the mountainous remote sensing images registration with land cover changes. And we will test the improved optical flow estimation algorithm on the large size. We should consider how to handle images with hybrid terrain including mountain area, farmland, urban areas and so on with large area of land cover changes in our future work as well.

\section{ACKNOWLEDGEMENTS}

The work was supported by the National Natural Science Foundation of China (NSFC) under Grant Nos. 61671334, 41701394.

\section{REFERENCES}

Alcantarilla, P. F., A. Bartoli and A. J. Davison 2012. KAZE Features. European Conference on Computer Vision (ECCV).

Bay, H., T. Tuytelaars and L. V. Gool 2006, "SURF: Speeded Up Robust Features," Computer Vision and Image Understanding, vol. 110, no. 3, pp. 404-417.

Black, M. J. and P. Anandan 1996, "The Robust Estimation of Multiple Motions: Parametric and Piecewise-Smooth Flow Fields," Computer Vision and Image Understanding, vol. 63, no. 1, pp. 75-104.

Brox, T., A. Bruhn, N. Papenberg and J. Weickert 2004. High Accuracy Optical Flow Estimation Based on a Theory for Warping. European Conference on Computer Vision (ECCV).

Feng, R., X. Li, W. Zou and H. Shen 2017. Registration of multitemporal GF-1 remote sensing images with weighting perspective transformation model. 2017 IEEE International Conference on Image Processing (ICIP).

Fischler, M. A. and R. C. Bolles 1981, "Random sample consensus: a paradigm for model fitting with applications to image analysis and automated cartography," Commun. ACM, vol. 24 , no. 6 , pp. 381-395.

Goshtasby, A. 1986, "Piecewise linear mapping functions for image registration," Pattern Recognition, vol. 19, no. 6, pp. 459-466.

Hel-Or, Y., H. Hel-Or and E. David 2011. Fast template matching in non-linear tone-mapped images. International Conference on Computer Vision (ICCV).

Horn, B. K. P. and B. G. Schunck 1981, "Determining optical flow," Artificial Intelligence, vol. 17, no. 1, pp. 185-203.

Johnson, K., A. Cole-Rhodes, I. Zavorin and J. Le Moigne 2001. Mutual information as a similarity measure for remote sensing image registration. Aerospace/Defense Sensing, Simulation, and Controls, International Society for Optics and Photonics.

Li, X., N. Hui, H. Shen, Y. Fu and L. Zhang 2015, "A robust mosaicking procedure for high spatial resolution remote sensing 
images," ISPRS Journal of Photogrammetry and Remote Sensing, vol. 109, pp. 108-125.

Lin, K., N. Jiang, S. Liu, L.-F. Cheong, M. Do and J. Lu 2017. Direct photometric alignment by mesh deformation. Proceedings of the IEEE Conference on Computer Vision and Pattern Recognition.

Liu, C., J. Yuen and A. Torralba 2011, "SIFT Flow: Dense Correspondence across Scenes and Its Applications," IEEE Transactions on Pattern Analysis and Machine Intelligence, vol. 33, no. 5, pp. 978-994.

Martinez, A., J. Garcia-Consuegra and F. Abad 1999. A correlation-symbolic approach to automatic remotely sensed image rectification. IEEE 1999 International Geoscience and Remote Sensing Symposium. IGARSS'99 (Cat. No.99CH36293).

Ren, Z., J. Li, S. Liu and B. Zeng 2017. Meshflow video denoising. 2017 IEEE International Conference on Image Processing (ICIP).

Sedaghat, A., M. Mokhtarzade and H. Ebadi 2011, "Uniform Robust Scale-Invariant Feature Matching for Optical Remote Sensing Images," IEEE Transactions on Geoscience and Remote Sensing, vol. 49, no. 11, pp. 4516-4527.
Shen, H., X. Meng and L. Zhang 2016, "An Integrated Framework for the Spatio-Temporal-Spectral Fusion of Remote Sensing Images," IEEE Transactions on Geoscience and Remote Sensing, vol. 54, no. 12, pp. 7135-7148.

Wang, Y., J. Shen, W. Liao and L. Zhou 2010. Automatic fundus images mosaic based on SIFT feature. 2010 3rd International Congress on Image and Signal Processing.

Wong, A. and D. A. Clausi 2007, "ARRSI: Automatic Registration of Remote-Sensing Images," IEEE Transactions on Geoscience and Remote Sensing, vol. 45, no. 5, pp. 1483-1493.

Yi, Z., C. Zhiguo and X. Yang 2008, "Multi-spectral remote image registration based on SIFT," Electronics Letters, vol. 44, no. 2, pp. 107-108.

Zagorchev, L. and A. Goshtasby 2006, "A comparative study of transformation functions for nonrigid image registration," IEEE Transactions on Image Processing, vol. 15, no. 3, pp. 529-538.

Zeng, C., H. Shen and L. Zhang 2013, "Recovering missing pixels for Landsat ETM+ SLC-off imagery using multitemporal regression analysis and a regularization method," Remote Sensing of Environment, vol. 131, pp. 182-194. 